УДК 531+539.3

\title{
СОУДАРЕНИЕ КЛИНА С ВЯЗКОУПРУГОЙ НИТЬЮ ПРИ ДОЗВУКОВОМ РЕЖИМЕ
}

\author{
Мамедов Тахир Джалил \\ доктор философии \\ Гянджинский Государственный Университет
}

Аннотация: В работе для дозвукового режима исследуются напряженное состояние и обрыв линейно вязкоупругой (типа Максвелла) нити при поперечном ударе жестким симметричным клином с постоянной скоростью.

Ключевые слова: Удар клином, вязкоупругая нить, коэффициенты, деформация, гибкая.

\section{COLLISION OF A WEDGE WITH A VISCOELASTIC THREAD IN SUBSONIC MODE}

\section{Mamedov Takhir Jalil}

\begin{abstract}
In the work tension and breakage of the linear viscoelastic (Maksvella type) thread are investigated for a subsonic mode at a cross blow by a rigid symmetric wedge with a constant speed.

Key words: Wedge impact, viscous elastic thread, coefficients, deformation, flexible.

Поведение гибкой связи при нормальном ударе твердым телом существенно зависит от физических свойств материала связи. В данной работе рассматривается напряженное состояние и обрыв линейной вязкоупругой нити, закон деформирования которой подчиняется уравнению Максвелла. Нормальный удар по гибкой нити производится симметричным жестким клином с постоянной скоростью.

Исследуется случай, когда в нити скорость точки излома меньше скорости звука (дозвуковой режим движения).
\end{abstract}




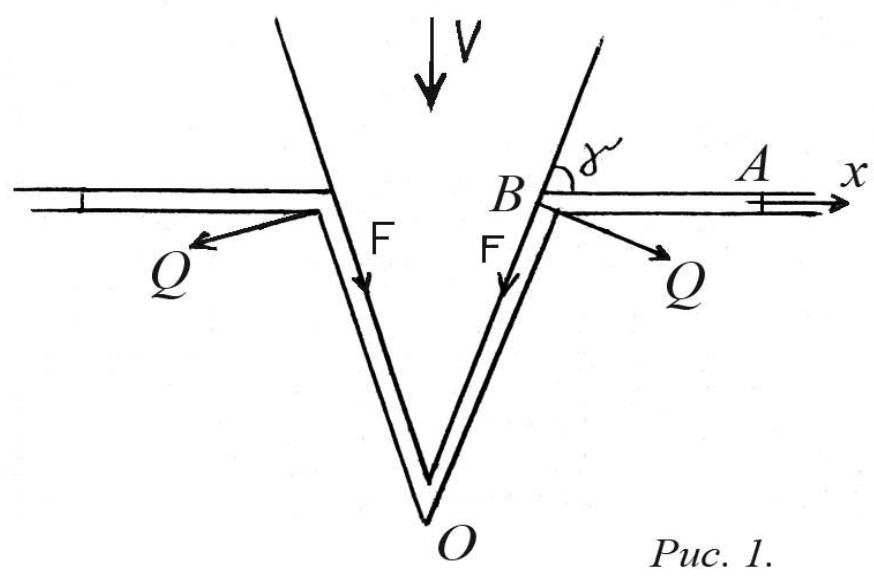

\$1. Пусть по бесконечно длинной гибкой прямолинейной ненапряженной гибкой нити производится нормальный удар жестким симметричным клином с постоянной скоростью $\mathrm{V}$ (рис.1). Принимается, что пригибная часть нити облегает щеку ударяющего клина.

В работе [2] экспериментально установлено, что при поперечном ударе клином разных углов раствора по резиновому шнуру, капроновым нитям разных диаметров $(d-0,9 m m-1,5 m m)$, наблюдается движение с полным облеганием.

Нужно определить напряженное состояние нити в областях за и перед точкой излома $B$ (рис.1), без и с учетом обрыва нити. Материал гибкой нити предполагается вязкоупругим

$$
\dot{\sigma} \frac{\mu}{E}+\sigma=\mu \dot{\varepsilon}
$$

или

$$
\dot{\sigma}+\frac{E}{\mu} \sigma=E \dot{\varepsilon}, \sigma=E\left[\varepsilon-k_{1} e^{-k_{1} t} \int_{0}^{t} e^{k_{1} \tau} \varepsilon(\tau, x) d \tau\right]
$$

Здесь $\sigma$ - напряжение; $\varepsilon$ - деформация; $\mu$ - коэффициент вязкости $(\mu=$ const $) ; \quad E$ - модуль Юнга; $k_{1}=\frac{E}{\mu}$ точки над буквами $\varepsilon$ и $\sigma$ означают производные по времени.

Уравнение движения нити в области облегания в области $O B$ и в области перед точкой излома $B$ (в области $B A$ ) соответственно, будут $[1,2]$ :

$$
\rho \frac{\partial^{2} u_{2}}{\partial t^{2}}=\frac{\partial \sigma}{\partial x}
$$


$\rho \frac{\partial^{2} u_{1}}{\partial t^{2}}=\frac{\partial \sigma}{\partial x}$

где $\rho$ - плотность; $t$ - время; $\sigma$ - напряжение; $u_{2}, x$ - эйлеровы и лагранжевы координаты частицы нити в области OB; $u_{1}$ - смещение частицы нити в области $B A$.

Учитывая закон деформирования (1.1) уравнения $(1.2),(1,3)$ соответственно примут вид

$$
\begin{aligned}
& \frac{1}{a_{0}^{2}} \frac{\partial^{2} u_{1}}{\partial t^{2}}=\frac{\partial^{2} u_{1}}{\partial x^{2}}-\frac{E}{\mu} \frac{\partial u_{1}}{\partial t} \\
& \frac{1}{a_{0}^{2}} \frac{\partial^{2} u_{2}}{\partial t^{2}}=\frac{\partial^{2} u_{2}}{\partial x^{2}}-\frac{E}{\mu} \frac{\partial u_{2}}{\partial t}
\end{aligned}
$$

Условие на волне сильного разрыва (в точке излома $B$ ) и закон деформирования в безразмерном виде будут $[1,2]$ :

$$
\begin{aligned}
& \frac{\bar{b}-\bar{v}_{1}}{1+\varepsilon_{1}}=\frac{b \sec \gamma-\bar{v}_{2}}{1+\varepsilon_{2}}=z^{-} \\
& \bar{z} \cdot\left(\bar{v}_{2}-\bar{v}_{1} \cos \gamma-M \sin \gamma\right)=\bar{\sigma}_{1} \cos \gamma-\bar{\sigma}_{2}-\bar{F} \\
& \bar{z} \cdot\left(M \cos \gamma-\bar{v}_{1} \sin \gamma\right)=\bar{Q}+\bar{\sigma}_{1} \sin \gamma \\
& \bar{\sigma}=\varepsilon-k e^{-k t} \int_{0}^{t} e^{k \tau} \varepsilon(\tau, x) d \tau
\end{aligned}
$$

Здесь

$$
\begin{aligned}
& \bar{b}=b a_{0}^{-1} ; b=v \operatorname{ctg} \gamma ; M=v a_{0}^{-1} ; \quad \bar{v}_{1}=v_{1} a_{0}^{-1} ; \bar{v}_{21}=v_{2} a_{0}^{-1} \\
& \bar{\sigma}_{1}=\sigma_{1} E^{-1} ; \bar{\sigma}_{2}=\sigma_{2} E^{-1} ; \quad \bar{F}=F \cdot E^{-1} ; \bar{Q}=Q \cdot E^{-1} ; \bar{z}=z \cdot a_{0}^{-1} ; \bar{\sigma}=\sigma E^{-1}
\end{aligned}
$$

Величины с черточкой - это безразмерные параметры; $\bar{z}$ - отношение скорости волны сильного разрыва к скорости упругой волны; $a_{0}^{2}=E \cdot \rho^{-1} ; \quad F$, $Q$ - касательная и нормальная к щеке клина, составляющая сосредоточенной силы, индексы 1, 2 обозначают величины перед и за точкой излома; $\gamma$ - угол между первоначальным положением нити и щекой клина.

Кроме этого, вводим безразмерные величины в виде

$$
\bar{u}_{2}=u_{2} \cdot R^{-1} ; \bar{u}_{1}=u_{1} \cdot R^{-1} ; \bar{x}=x \cdot R^{-1} ; \bar{t}=a_{0} t R^{-1} ; \bar{k}=\frac{E}{\mu} \frac{R}{a_{0}}
$$

$R$-постоянная величина, имеющая размерность длины (радиус кругового поперечного сечения нити).

В дальнейшем во всех выражениях черточки над буквами опускаем. 
Уравнения движения (1.4) и (1.5) с учетом (1.9) в безразмерном виде будут

$$
\begin{aligned}
& \frac{\partial^{2} u_{2}}{\partial t^{2}}=\frac{\partial^{2} u_{2}}{\partial x^{2}}-k \frac{\partial u_{2}}{\partial t} \\
& \frac{\partial^{2} u_{1}}{\partial t^{2}}=\frac{\partial^{2} u_{1}}{\partial x^{2}}-k \frac{\partial u_{1}}{\partial t}
\end{aligned}
$$

Деформации $\varepsilon_{1}, \varepsilon_{2}$ за и перед фронтом волны сильного разрыва выражаются в виде

$$
\varepsilon_{2}=\frac{\partial u_{2}}{\partial x}-1 ; \varepsilon_{1}=\frac{\partial u_{1}}{\partial x}
$$

Имеем условие в точке удара (без обрыва нити)

$$
\left.u_{2}(x, t)\right|_{x=0}=0
$$

и условие на фронте упругой волны (в точке А)

$$
\left.u_{1}(x, t)\right|_{x=t}=0
$$

\$2. Для получения решения задачи в области $O B(0 \leq x<z t)$ и ВА $(z t \leq x<t)$ функции $u_{n}$ представим в виде разложения по параметру $k$

$$
u_{n}(x, t)=\sum_{i=0}^{\infty} k^{i} u_{n}^{(i)}
$$

Здесь $n=1,2, u_{2}$ - относится к области, ОВ, а $u_{1}$ - к области ВА (рис.1), $k$ считается малым, т.е.. $k<1$.

Ограничимся двумя членами в правой части (2.1) и решение задачи в каждой области представим в виде

$$
\begin{aligned}
& u_{2}(x, t)=u_{2}^{0}(x, t)+k u_{2}^{(1)}(x, t) \\
& u_{1}(x, t)=u_{1}^{0}(x, t)+k u_{1}^{(1)}(x, t)
\end{aligned}
$$

где

$$
\left.\begin{array}{l}
u_{2}^{0}=a_{02}(t-x)+b_{02}(t+x) ; \\
u_{2}^{(1)}=a_{22}(t-x)^{2}+b_{22}(t+x)^{2}-\frac{a_{02}+b_{02}}{4}\left(t^{2}-x^{2}\right) \\
u_{1}^{0}=a_{01}(t-x)+b_{01}(t+x) ; \\
u_{1}^{(1)}=a_{11}(t-x)^{2}+b_{11}(t+x)^{2}-\frac{a_{01}+b_{01}}{4}\left(t^{2}-x^{2}\right)
\end{array}\right\}
$$


Здесь, $u_{2}^{(0)}, u_{1}^{(0)}$ - решения задачи соответствуют линейно упругому случаю; $a_{02}, b_{02}, a_{22}, b_{22}, a_{01}, b_{01}, a_{11}, b_{11}$ - пока неизвестные коэффициенты и определяются

из краевых условий (1.6), (1.7), (1.8), (1.12), (1.13).

Траекторию волны сильного разрыва представим в виде разложения по параметру $k$.

$$
x_{*}(t)=z_{0} t+k z_{1} t^{2}+\ldots
$$

Тогда скорость волны сильного разрыва будет

$$
z=\frac{d x_{*}}{d t}=z_{0}+k 2 z_{1} t
$$

В формуле (2.6) все величины безразмерные; $z_{0}, z_{1}$, - неизвестные пока константы. Здесь ограничимся случаем двух членов в правой части (2.6).

Сначала, построим решение задачи в случае, когда выполняется неравенство [2]

$$
F<\mu_{*} Q
$$

Неравенство (2.8) влечет за собой условие прилипания частицы нити за точкой излома, т.е. в точке излома относительная скорость частицы нити равна нулю

$$
v_{2}=\frac{\partial u_{2}}{\partial t}=0
$$

Подставляя (2.2)-(2.5), в условиях (1.6), (1.12), (1.13), (2.9), с учетом (2.6), (2.7) и, приравнивая коэффициенты при одинаковых степенях $k$ определяем неизвестные константы в виде:

$$
\begin{aligned}
& a_{02}=-b_{02} ; 2 b_{02}-1=b \cdot(\sec \gamma-1)=\varepsilon_{2}^{(0)} ; b=M \cdot \operatorname{ctg} \gamma ; \\
& b_{22}=0 ;-a_{01}=b \cdot(\sec \gamma-1)=\varepsilon_{1}^{(0)} ; a_{22}=0 \\
& b_{01}=0 ; b_{11}=0 ; a_{11}=\frac{a_{01}}{4} \frac{1+z_{0}}{1-z_{0}} ; z_{1}=0 \\
& z_{0}=\frac{b}{b+(1-b) \cos \gamma} ; v_{2}=\frac{\partial u_{2}}{d t}=v_{2}^{(0)}=0
\end{aligned}
$$

Таким образом, решение задачи в случае $F<\mu_{*} Q$ в каждой области, т.е.в области за и перед точкой излома в соответственно определяются в виде: 


$$
\begin{aligned}
& u_{1}(x, t)=\varepsilon_{1}^{0}(x-t)-\frac{k \varepsilon_{1}^{0}}{4}\left[\frac{1+z_{0}}{1-z_{0}}(t-x)^{2}+\left(t^{2}-x^{2}\right)\right] ; z_{0} t \leq x \leq 1 \\
& u_{2}(x, t)=\left(1+\varepsilon_{2}^{0}\right) x ; 0 \leq x \leq z_{0} t
\end{aligned}
$$

Следовательно, деформации, скорость частицы и напряжения в области ОВ и ВА, соответственно будут:

$$
\begin{aligned}
& \left\{\begin{array}{l}
\varepsilon_{1}=\varepsilon_{1}^{(0)}\left\{1+\frac{k}{2}\left[\frac{1+z_{0}}{1-z_{0}}(t-x)+x\right]\right\} \\
v_{1}=\varepsilon_{1}^{(0)}\left\{-1-\frac{k}{2}\left[\frac{1+z_{0}}{1-z_{0}}(t-x)+t\right]\right\}
\end{array}\right. \\
& \left\{\begin{array}{c}
\sigma_{1}=\varepsilon_{1}^{(0)}\left\{1+\frac{k}{1}\left[\frac{1+z_{0}}{1-z_{0}}(t-x)-x+2 t\right]\right. \\
\varepsilon_{2}=\varepsilon_{2}^{(0)}=b(\sec \gamma-1)=\varepsilon_{1}^{(0)}, \\
v_{2}=\frac{\partial u_{2}}{\partial t}=v_{2}^{(0)}=0, \\
\sigma_{2}=\varepsilon_{2}^{(0)}(1-k t), \\
z_{0}=b[b+(1-b) \cos \gamma]^{-1}
\end{array}\right\}
\end{aligned}
$$

Здесь $\varepsilon_{1}^{0}, \varepsilon_{2}^{(0)}, v_{2}^{(0)}, z^{(0)}$ соответствуют деформации, скорость частиц, скорость волны сильного разрыва упругой задачи. Здесь и далее индекс 2 относится к области ОВ, а индекс 1 относится к области ВА.

Теперь определим решение задачи при $\gamma>2 \gamma_{*}$. При этом выполняется условие [2]

$$
F=\mu_{*} Q
$$

Решения уравнений (1.9), (1.10) для этого случая представим в виде (2.2) $-(2.5)$

Из условий (1.12), (1.13) определим следующие неизвестные константы в виде

$$
b_{01}=b_{11}=0 ; b_{02}=-a_{02} ; \quad b_{22}=-a_{22}
$$

Учитывая (2.15), величины, $\varepsilon_{1}, v_{1}, \sigma_{1}, \varepsilon_{2}, v_{2}, \sigma_{2}$ принимают следующий вид

$$
\left.\begin{array}{l}
\varepsilon_{1}=-a_{01}+k\left[-2 a_{11}(t-x)+\frac{a_{01}}{2} x\right] ; \\
v_{1}=a_{01}+k\left[2 a_{11}(t-x)+\frac{a_{01}}{2} t\right] ; \\
\sigma_{1}=-a_{01}+k\left[-2 a_{11}(t-x)+\frac{a_{01}}{2}(x+2 t)\right] ;
\end{array}\right\}
$$




$$
\left.\begin{array}{l}
\varepsilon_{2}=2 b_{02}-1+k 4 b_{22} t ; v_{2}=k 4 b_{22} x ; \\
\sigma_{2}=2 b_{02}-1+k\left[4 b_{22}-\left(2 b_{02}-1\right) t\right] ;
\end{array}\right\}
$$

Далее, подставляя (2.16), (2.17), (2.6), (2.7) в условия (1.6), (1.7), (1.8) с учетом (2.14) и приравнивая коэффициенты при одинаковых степенях $k$, определяем неизвестные константы $a_{01}, a_{11}, b_{02}, b_{22}, z_{1}$ в виде

$$
\begin{aligned}
& a_{01}=-\frac{z_{0}-b}{1-z_{0}} ; b_{02}=\frac{b}{z_{0}} \sec \gamma \\
& a_{11}=\frac{a_{01}}{4} \frac{1+\mathrm{z}_{0}}{1-\mathrm{z}_{0}}-\frac{z_{1}}{\left(1-z_{0}\right)^{2}}\left(1-a_{01}\right) ; \mathrm{b}_{22}=\frac{-z_{1} b_{02}}{z_{0} 2}>0 ; \\
& z_{1}=\frac{\varphi_{1}\left(\varepsilon_{1}^{(0)}, \varepsilon_{2}^{(0)}, z_{0}, \gamma, \mu_{*}\right)}{\varphi_{2}\left(M, \gamma, \gamma_{*}, z_{0}, \varepsilon_{2}^{(0)}\right)}<0 \\
& \varphi_{2}=2\left[M \cos \gamma\left(\operatorname{tg} \gamma-\operatorname{tg} \gamma_{*}\right)+\left(1+\operatorname{tg} \gamma_{*} \operatorname{tg} \gamma\right) \cos \gamma+\frac{1+z_{0}^{2}}{z_{0}} \frac{1+\varepsilon_{2}^{(0)}}{2}\right]>0 ; \\
& \varphi_{1}=-\varepsilon_{2}^{(0)}+\varepsilon_{1}^{(0)}\left(1+\operatorname{tg} \gamma \operatorname{tg} \gamma_{*}\right)\left(1-\frac{1+z_{0}^{2}}{2}\right) \cos \gamma<0 ;
\end{aligned}
$$

Константа $z_{0}$ определяется из следующего уравнения

$b \cdot \varphi_{3}=\varphi_{4}$

Здесь

$$
\begin{aligned}
& \varphi_{3}=z_{0} \operatorname{tg} \gamma_{*} \sin \gamma \cos \gamma+z_{0}^{2} \operatorname{tg} \gamma_{*} \sin \gamma+1+z_{0} \cos ^{2} \gamma-z_{0}^{2} \sin ^{2} \gamma ; \\
& \varphi_{4}=z_{0} \cos \gamma+z_{0}^{2} \cos ^{2} \gamma+z_{0}^{2} \operatorname{tg} \gamma_{*} \sin \gamma \cos \gamma
\end{aligned}
$$

Как видно, решить уравнение (2.19) относительно $z_{0}$ не удобно, но оно легко разрешается относительно $b$ и при заданном $z_{0}$ :

$$
b=\varphi_{4} \cdot \varphi_{3}^{-1}
$$

Таким образом, определены все неизвестные коэффициенты, и основные параметры задачи $\varepsilon_{1}, v_{1}, \sigma_{1}, \varepsilon_{2}, v_{2}, \sigma_{2}$ выражаются формулами

$$
\begin{aligned}
& \varepsilon_{1}=\varepsilon_{1}^{0}+k \varepsilon_{1}^{(0)}\left\{\left[\frac{1+z_{0}}{4\left(1-z_{0}\right)}+\frac{z_{1}}{\left(1-z_{0}\right)^{2}}\left(1+\frac{1}{\varepsilon_{1}^{(0)}}\right)\right](t-x)-\frac{x}{2}\right\} ; \\
& v_{1}=v_{1}^{0}+k v_{1}^{(0)}\left\{\left[\frac{1+z_{0}}{4\left(1-z_{0}\right)}+\frac{z_{1}}{\left(1-z_{0}\right)^{2}}\left(\frac{1}{v_{1}^{(0)}}-1\right)\right](t-x)+\frac{t}{2}\right\} ; \\
& \sigma_{1}=\sigma_{1}^{0}+k \sigma_{1}^{(0)}\left\{\left[\frac{1+z_{0}}{4\left(1-z_{0}\right)}+\frac{z_{1}}{\left(1-z_{0}\right)^{2}}\left(1+\frac{1}{\sigma_{1}^{(0)}}\right)\right](t-x)-\frac{x+2 t}{2}\right\} ;
\end{aligned}
$$




$$
\begin{aligned}
& \varepsilon_{2}=\varepsilon_{2}^{(0)}-k \frac{z_{1}}{z_{0}}\left(1+\varepsilon_{2}^{(0)}\right) t ; \quad v_{2}=-k \frac{z_{1}}{z_{0}}\left(1+\varepsilon_{2}^{(0)}\right) x>0 ;\left(z_{1}<0\right) ; \\
& \sigma_{2}=\sigma_{2}^{(0)}-k\left[\frac{z_{1}}{z_{0}}\left(1+\varepsilon_{2}^{(0)}\right)+\varepsilon_{2}^{(0)}\right] t ; \quad v_{2}=0 ;
\end{aligned}
$$

Из формулы (2.22) следует, что скорость частицы нити $v_{2}$ в области $0 \leq x \leq x_{*}(t)$ равна нулю только в точке удара $(x=0)$ и при $0<x<x_{*}(t)$ растет по переменной $x$. Из формулы (2.18), (2.7) следует, что скорость фронта волны сильного разрыва в вязкоупругой нити меньше, чем в упругой нити.

Здесь $\sigma_{2}^{(0)}=\varepsilon_{2}^{(0)} ; \sigma_{1}^{(0)}=\varepsilon_{1}^{(0)} ; \varepsilon_{1}^{(0)}, \varepsilon_{2}^{(0)}, v_{1}^{(0)}, v_{2}^{(0)}$ деформации и скорость частиц линейно упругой нити. Если не учитывать вязкостные свойства материала нити, то из формулы решения линейно упругой задачи, они выражаются в виде.

$$
\begin{aligned}
& \varepsilon_{1}^{(0)}=\frac{z_{0}}{1-z_{0}} \frac{1-\cos \gamma-z_{0}^{2} \cdot \alpha_{2} \cdot 0,5 \sin 2 \gamma}{1+z_{0} \cdot \alpha_{1} \cdot \cos ^{2} \gamma-0,5 \cdot z_{0}^{2} \cdot \alpha_{2} \cdot \sin 2 \gamma} ; \quad \sigma_{1}^{(0)}=\varepsilon_{1}^{(0)} ; \\
& \varepsilon_{2}^{(0)}=z_{0} \cos \gamma \frac{z_{0} \cdot \alpha_{2} \cdot \sin \gamma-\alpha_{2}(1-\cos \gamma)}{1+z_{0} \cdot \alpha_{1} \cdot \cos ^{2} \gamma-0,5 \cdot z_{0}^{2} \cdot \alpha_{2} \cdot \sin 2 \gamma} ; \quad \sigma_{2}^{(0)}=\varepsilon_{2}^{(0)} \\
& v_{1}^{(0)}=-\varepsilon_{1}^{(0)} ; \alpha_{1}=1+\operatorname{tg} \gamma \cdot \operatorname{tg} \gamma_{*} ; \quad v_{2}^{(0)}=0, \alpha_{2}=\operatorname{tg} \gamma-\operatorname{tg} \gamma_{*}
\end{aligned}
$$

§ 3. Теперь построим решение данной задачи с учетом обрыва нити. Для этого определяется $\max \sigma$ и эта величина приравнивается критическому (разрушающему) напряжению $\sigma_{k p}$. Здесь $\sigma_{k p}$ - прочность на разрыв материала нити.

Исследуем обрыв вязкоупругой нити в случае когда на волне сильного разрыва ( в точке излома В) имеет место условие (2.8), т.е. условие (2.9). Из формулы (2.13) следует, что напряжение в точке удара $(x=0)$ больше тем в области $0<x \leq x_{*}(t)$. Поэтому предельное условие должно быть в качестве $\max \sigma$, это условие в точке удара $(x=0)$ примет вид

$$
2 \varepsilon_{2}^{(0)}(1-k t)=\sigma_{n p}^{(\hat{a})}
$$

Здесь $\sigma_{n p}^{(b)}=\frac{\bar{\sigma}_{n p}^{(\hat{a})}}{\rho a_{0}^{2}} ; \bar{\sigma}_{n p}^{(\hat{a})}$ прочность на разрыв вязкоупругой нити,

$$
2 \varepsilon_{2}^{(0)}=2 \sigma_{2}^{(0)}=\sigma_{n p}^{(y)} ; \sigma_{n p}^{(y)}=\frac{\bar{\sigma}_{n p}^{(y)}}{\rho a_{0}^{2}} ;
$$

где $\bar{\sigma}_{n p}^{(y)}$-прочность на разрыв упругой нити; $\varepsilon_{2}^{(0)}, \sigma_{2}^{(0)}$ выражаются формулами (2.13). Из формулы (3.1) получим 


$$
\sigma_{n p}^{(y)}(1-k t)=\sigma_{n p}^{(\hat{a})}
$$

Из выражения (3.2) следует, что при $\sigma_{n p}^{(y)}=\sigma_{n p}^{(\hat{a})}$ вязкоупругая нить обрывается в точке удара в момент удара (рис. 2), а при $\sigma_{n p}^{(y)}<\sigma_{n p}^{(\hat{a})}$ нить не обрывается.

При $\sigma_{i \partial}^{(0)}>\sigma_{i \partial}^{(b)}$ вязкоупругая нить обрывается.

Для решения задачи с обрывом при $\gamma<2 \gamma_{*}$ нужно решить уравнение движения нити (1.9), (1.10) с условиями (1.6) - (1.8), (1.13), (2.14) и с учетом условия

$$
\left.\sigma_{2}(x, t)\right|_{x=0}=0
$$

учитывающим факт обрыва нити в точке удара $x=0$.

Решение задачи в области облегания нити к щеке клина (рис.2) и в области перед точкой налегания $B$ представляются в виде (2.2) - (2.5). Подставляя (2.2) - (2.5) в условиях (1.6) - (1.8), (1.13), (2.14), (3.3) с учетом (2.6), (2.7) и приравнивая коэффициенты при одинаковых степенях $k$, определяем неизвестные константы $a_{01}, b_{01}, a_{11}, b_{11}, a_{02}, b_{02}, a_{22}, b_{22}, z_{0}, z_{1}$ в виде

$$
\begin{aligned}
& b_{02}=1+a_{02} ; \quad b_{22}=a_{22} ; a_{01}=-\frac{z_{0}-b}{1-z_{0}}=-\varepsilon_{1}^{(0)} \\
& 2 a_{02}+1=b \sec \gamma-z_{0} ; b_{01}=0 ; b_{11}=0 ; a_{11}=\frac{a_{01}}{4} \frac{1+z_{0}}{1-z_{0}}-\frac{1-a_{01}}{\left(1-z_{0}\right)^{2}} z_{1} ; \\
& b_{22}=-\frac{z_{1}}{\left(1-z_{0}^{2}\right)} \frac{1}{2}+\frac{2 a_{02}+1}{8} \frac{1-z_{0}^{2}}{1-z_{0}^{2}} ; \\
& b=z_{0} \cos \gamma \frac{z_{0}+\alpha_{1} \cos \gamma}{z_{0}+\alpha_{1} \cdot \cos ^{2} \gamma-0,5 \cdot \alpha_{2} \cdot z_{0} \cos 2 \gamma} ; 2 a_{02}+1=v_{2}^{(0)} \\
& v_{2}^{(0)}=z_{0} \cos \gamma \frac{\left(1+\alpha_{1}\right)(1-\cos \gamma)+\alpha_{2} \cdot z_{0} \sin \gamma}{z_{0}+\alpha_{1} \cdot \cos ^{2} \gamma+0,5 \cdot \alpha_{1} \cdot z_{0} \cdot \sin 2 \gamma}>0 \\
& \varepsilon_{1}^{(0)}=\frac{z_{0}}{1-z_{0}^{2}} \frac{\sin \gamma\left(\operatorname{tg}_{*}-\operatorname{tg} \frac{\gamma}{2}\right)}{\left(1+\alpha_{1}\right)\left(1+z_{0}\right) \cos \gamma}>0 \\
& \gamma<2 \gamma_{*}, v_{1}^{(0)}=-\varepsilon_{1}^{(0)}, \sigma_{2}^{(0)}=\varepsilon_{2}^{(0)}=0 \\
& z_{1}=-\frac{\psi_{1}}{\psi_{2}}<0 ;
\end{aligned}
$$




$$
\begin{aligned}
& \psi_{1}=\left(1+\mu_{*} \operatorname{tg} \gamma\right) \varepsilon_{1}^{(0)} \cos \gamma+v_{2}^{(0)} z_{0} \frac{1-z_{0}^{2}}{1+z_{0}^{2}}-\frac{\varepsilon_{1}^{(0)}}{2}\left[\left(1-z_{0}^{2}\right) \mu_{*} \sin \gamma+\cos \gamma-z_{0}\right] \\
& \psi_{2}=2\left\{v_{0}^{2}+b \sin \gamma\left(\mu_{*}+\operatorname{tg} \gamma\right)+\varepsilon_{1}^{(0)}\left(1-\mu_{*} \operatorname{tg} \gamma\right) \cos \gamma+\frac{1+\varepsilon_{1}^{(0)}}{1-z_{0}}\left(z_{0}-\cos \gamma\right)-\frac{2 z_{0}}{1+z_{0}^{2}}\right\}
\end{aligned}
$$

В области $x_{*}(t) \leq x \leq t$ деформация $\varepsilon_{1}$, скорость частиц $v_{1}$ и напряжения $\sigma_{1}$ определяются в виде (2.16), но $a_{11}, \varepsilon_{1}^{(0)}, z_{0}, z_{1}$ выражаются формулами (3.4), (3.7), (3.5), (3.8) соответственно.

В области от точки обрыва $(x=0)$ до фронта волны сильного разрыва $\left(0 \leq x \leq x_{*}(t)\right)$ деформация $\varepsilon_{2}$, скорость $v_{2}$ и напряжения $\sigma_{2}$ определяются в виде

$$
\varepsilon_{2}=\frac{k}{1+z_{0}^{2}}\left(v_{2}^{(0)}-2 z_{1}\right) x ; v_{2}=v_{2}^{(0)}-\frac{k}{1+z_{0}^{2}}\left(z_{0} v_{2}^{(0)}+z_{1}\right) t
$$

$$
\sigma_{2}=\frac{k}{1+z_{0}^{2}}\left(v_{2}^{(0)}-2 z_{1}\right) x
$$

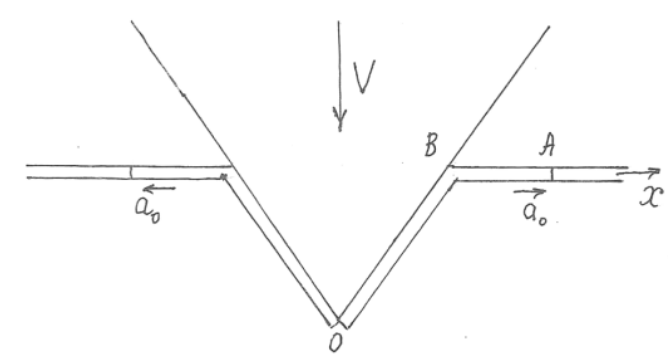

Рис. 2.

Отметим, что при определении напряжения по формуле (1.1) функция $e^{-k t}$ разложена в ряд Маклорена и ограничимся двумя членами этого разложения.

Из формулы (3.10) следует, что вязкоупругая нить за волной сильного разрыва напряжения деформация равна нулю только в точке $(x=0)$ обрыва и принимают положительные значения в области $0<x \leq x_{*}(t)$. Отметим что при линейно упругой задачи, в области облеганя ОВ (рис. 2) нить свободна от напряжений [2] 


\section{Список литературы}

1. Рахматуллин Х.А., Демьянов Ю.А. Прочность при интенсивных кратковременных нагрузках. Физматиз. М., 1961, 399с.

2. Муталлимов Ш.М. Волновая динамика гибких связей. Издательство “Elm”, Баку, 2001, 267 c. 